\title{
Assessment of Renal Toxicity Associated with Vancomycin in Patients with Health Care-Associated Methicillin-Resistant Staphylococcus Aureus Pneumonia: A Retrospective Analysis
}

\author{
Anand Kumar Singh ${ }^{1 *}$, Poonam Verma ${ }^{2}$ \\ ${ }^{1 *}$ Associate Professor, 2 Professor, Department of Physiology, \\ Shri Guru Ram Rai Institute of Medical and Health Sciences, Dehradun, Uttarakhand, India
}

\begin{abstract}
Background: One of the major causes of serious infections in both community and institutional settings is Staphylococcus aureus, with methicillin-resistant strains causing numerous invasive infections, especially those involving the bloodstream. Some practitioners have extrapolated these dosing recommendations with associated trough concentration goals to all vancomycin use. However, evidence of superior efficacy is lacking, and results of several single-centers, mostly retrospective trials have suggested that this more aggressive dosing may be related to an increased incidence of vancomycin-related nephrotoxicity and ototoxicity. Therefore, we retrospectively analyzed the rate of renal toxicity in patients treated with vancomycin for MRSA health care-associated pneumonia confirmed by bronchoalveolar lavage cultures.

Materials \& Methods: The present study was carried out in the department of pharmacology and renal medicine and included all the patients with MRSA HCAP, microbiologically confirmed by BAL semiquantitative cultures, who were treated with vancomycin were eligible for this investigation. Patients with polymicrobial infection demonstrated by BAL cultures, those treated with vancomycin for $<72$ hours, and those with acute renal failure or requiring dialysis were excluded from evaluation. For all study patients, the following information was recorded by one of the investigators; age, sex, weight, serial serum creatinine ( $\mathrm{SCr}$ ) measurements, age- and sex-adjusted creatinine clearance $(\mathrm{CrCl})$ and other parameters. Patients must have received the potentially renal toxic medication(s) while on vancomycin and before the maximum documented $\mathrm{SCr}$ (SCrmax) during treatment. Vasopressor use was considered when it was initiated or maintained within 48 hours before SCrmax. All the results were analyzed by SPSS software. Chi-square test was used for the assessment of level of significance.
\end{abstract}

\section{INTRODUCTION}

One of the major cause of serious infections in both community and institutional settings is Staphylococcus aureus, with methicillin-resistant strains causing numerous invasive infections, especially those involving the bloodstream. ${ }^{1}$ Due to concerns with efficacy as they relate to adequacy of dosing and related plasma concentrations, it is currently commonplace to administer vancomycin in doses intended to achieve trough concentrations of
Results: Mean age of the patients in group without and with renal toxicity was 58.2 and 61.2 years respectively. The mean male's percentage in the patients of the group without renal toxicity and with renal toxicity was 58.2 and 69.2 respectively. $71.5 \%$ and $81.7 \%$ were the mean percentage of whites in the group without and with renal toxicity. Mean APACHE II score in patients without and with renal toxicity was 18.6 and 23.1 respectively. Non-significant results were obtained while comparing the two study groups for the mean serum creatinine concentration and mean creatinine clearance. Significant correlation was obtained while comparing the vancomycin serum trough serum conc. $(\mu \mathrm{g} / \mathrm{mL})$ and percentage of renal toxicity.

Conclusion: Among patients treated for MRSA HCAP, aggressive vancomycin dosing and prolonged administration of vancomycin may be associated with renal toxicity.

Key Words: Renal, Staphylococcus, Vancomycin.

\section{*Correspondence to:}

Dr Anand Kumar Singh,

Associate Professor, Department of Physiology,

Shri Guru Ram Rai Institute of Medical and Health Sciences, Dehradun, Uttarakhand, India.

\section{Article History:}

Received: 28-08-2016, Revised: 14-09-2016, Accepted: 26-09-2016

\begin{tabular}{|l|r|}
\hline \multicolumn{2}{|c|}{ Access this article online } \\
\hline $\begin{array}{l}\text { Website: } \\
\text { www.ijmrp.com }\end{array}$ & Quick Response code \\
\hline $\begin{array}{l}\text { DOl: } \\
\text { 10.21276/ijmrp.2016.2.5.040 }\end{array}$
\end{tabular}

$15 \mathrm{mg} /$ liter or higher in the treatment of methicillin-resistant $\mathrm{S}$. aureus (MRSA) infections. ${ }^{2,3}$ In 2009, a consensus paper published recommended that patients be dosed with this end in mind. Some practitioners have extrapolated these dosing recommendations with associated trough concentration goals to all vancomycin use. However, evidence of superior efficacy is lacking, and results of several single-center, mostly retrospective 
trials have suggested that this more aggressive dosing may be related to an increased incidence of vancomycin-related nephrotoxicity and ototoxicity. ${ }^{4-6}$ Therefore, we retrospectively analyzed the rate of renal toxicity in patients treated with vancomycin for MRSA health care-associated pneumonia (HCAP) confirmed by bronchoalveolar lavage (BAL) cultures.

\section{MATERIALS \& METHODS}

The present study was carried out in the department of pharmacology and renal medicine and included all the patients with MRSA HCAP, microbiologically confirmed by BAL semiquantitative cultures, who were treated with vancomycin were eligible for this investigation. Patients with polymicrobial infection demonstrated by BAL cultures, those treated with vancomycin for $<72$ hours, and those with acute renal failure or requiring dialysis were excluded from evaluation. Ethical approval was taken from the ethical committee of the institution and written consent was obtained after explaining them the entire research protocol. For the assessment of the effect of vancomycin serum trough concentrations on the occurrence of renal toxicity, a retrospective study was planned. Per protocol, all patients were initially treated with weight-based vancomycin, receiving $30 \mathrm{mg} / \mathrm{kg}$ in 2 divided doses during a 24-hour period. To identify potential study patients, a computerized list of all patients with MRSA HCAP who had received vancomycin and had available through concentrations was generated by the Department of Medicine through retrospective query of the microbiology, laboratory, and pharmacy databases. For all study patients, the following information was recorded by one of the investigators (M.N.J.): age, sex, weight, serial serum creatinine $(\mathrm{SCr})$ measurements, age- and sexadjusted creatinine clearance $(\mathrm{CrCl})$ calculated during receipt of vancomycin, measured vancomycin serum trough concentrations, duration of vancomycin therapy, and Acute Physiology and Chronic Health Evaluation (APACHE) II scores. Patients must have received the potentially renal toxic medication(s) while on vancomycin and before the maximum documented SCr (SCrmax) during treatment. Vasopressor use was considered when it was initiated or maintained within 48 hours before SCrmax. All the results were analyzed by SPSS software. Chi-square test was used for the assessment of level of significance.

Graph 1: Demographic details of the patients

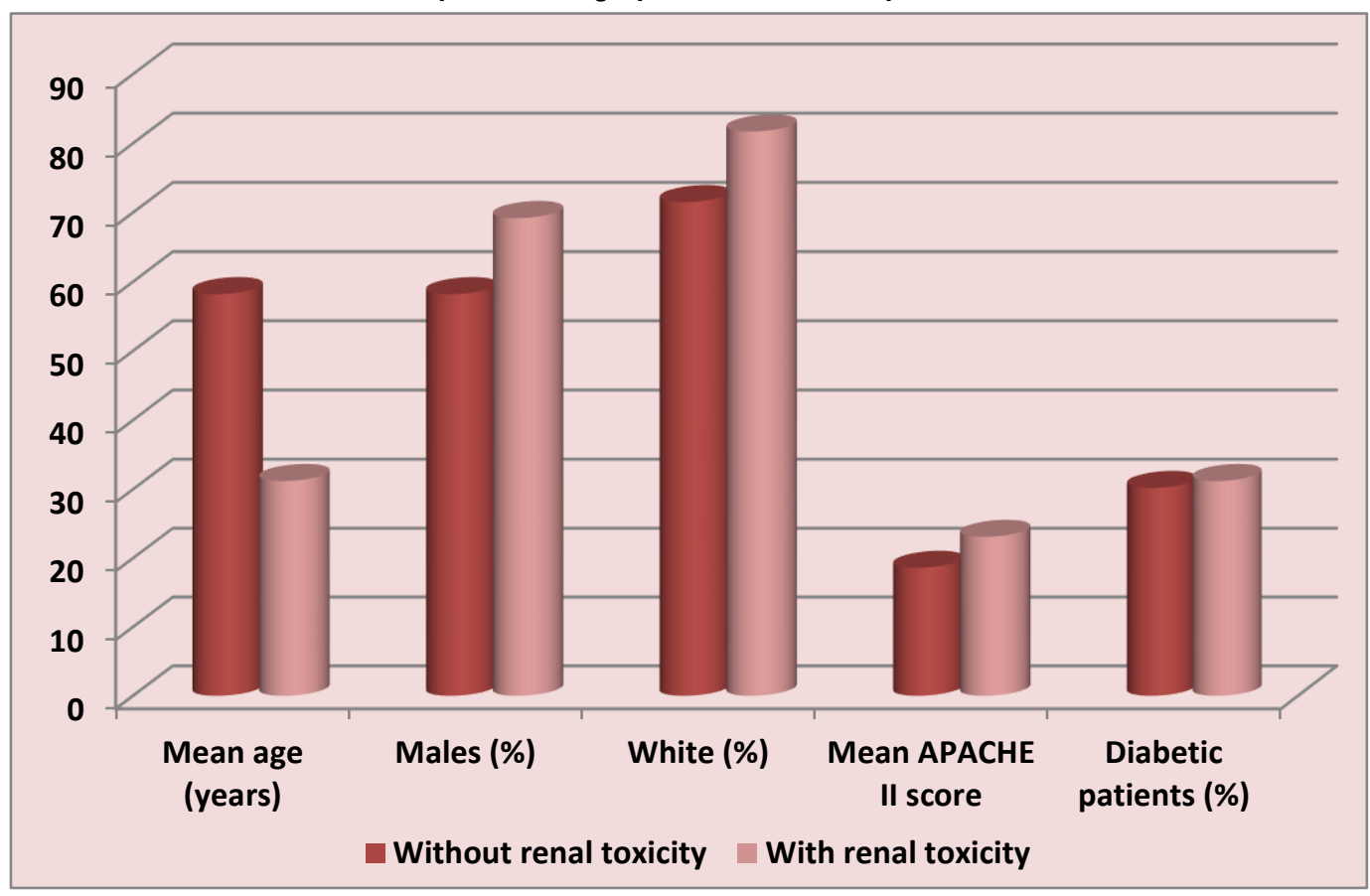

Table 1: $p$-value for the demographic details of the patients

\begin{tabular}{lccc}
\hline Parameter & Without renal toxicity & With renal toxicity & p-value \\
\hline Mean age (years) & 58.2 & 61.2 & 0.415 \\
Males (\%) & 58.2 & 69.2 & 0.652 \\
White (\%) & 71.5 & 81.7 & 0.125 \\
Mean APACHE II score & 18.6 & 23.1 & $0.001^{*}$ \\
Diabetic patients (\%) & 30.2 & 31.2 & 0.715 \\
\hline
\end{tabular}

*: Significant, APACHE: Acute Physiology and Chronic Health Evaluation

\section{RESULTS}

Graph 1 shows the demographic details of the patients in the two study groups. Mean age of the patients in group without and with renal toxicity was 58.2 and 61.2 years respectively. The mean male's percentage in the patients of the group without renal toxicity and with renal toxicity was 58.2 and 69.2 respectively. 71.5
$\%$ and $81.7 \%$ were the mean percentage of whites in the group without and with renal toxicity. Mean APACHE II score in patients without and with renal toxicity was 18.6 and 23.1 respectively. Table 1 shows the $p$-value for the demographic details of the patients. Significant correlation was obtained while comparing the 
mean APACHE II score in the patients of the two study groups. Graph 2 shows the baseline renal details of the patients. Table 2 shows the p-value for the baseline renal details of the patients. Non-significant results were obtained while comparing the two study groups for the mean serum creatinine concentration and mean creatinine clearance. Table 3 shows the occurrence of renal toxicity, stratified by vancomycin steady-state serum trough concentration. Significant correlation was obtained while comparing the vancomycin serum trough serum conc. $(\mu \mathrm{g} / \mathrm{mL})$ and percentage of renal toxicity.

Graph 2: Baseline renal details of the patients

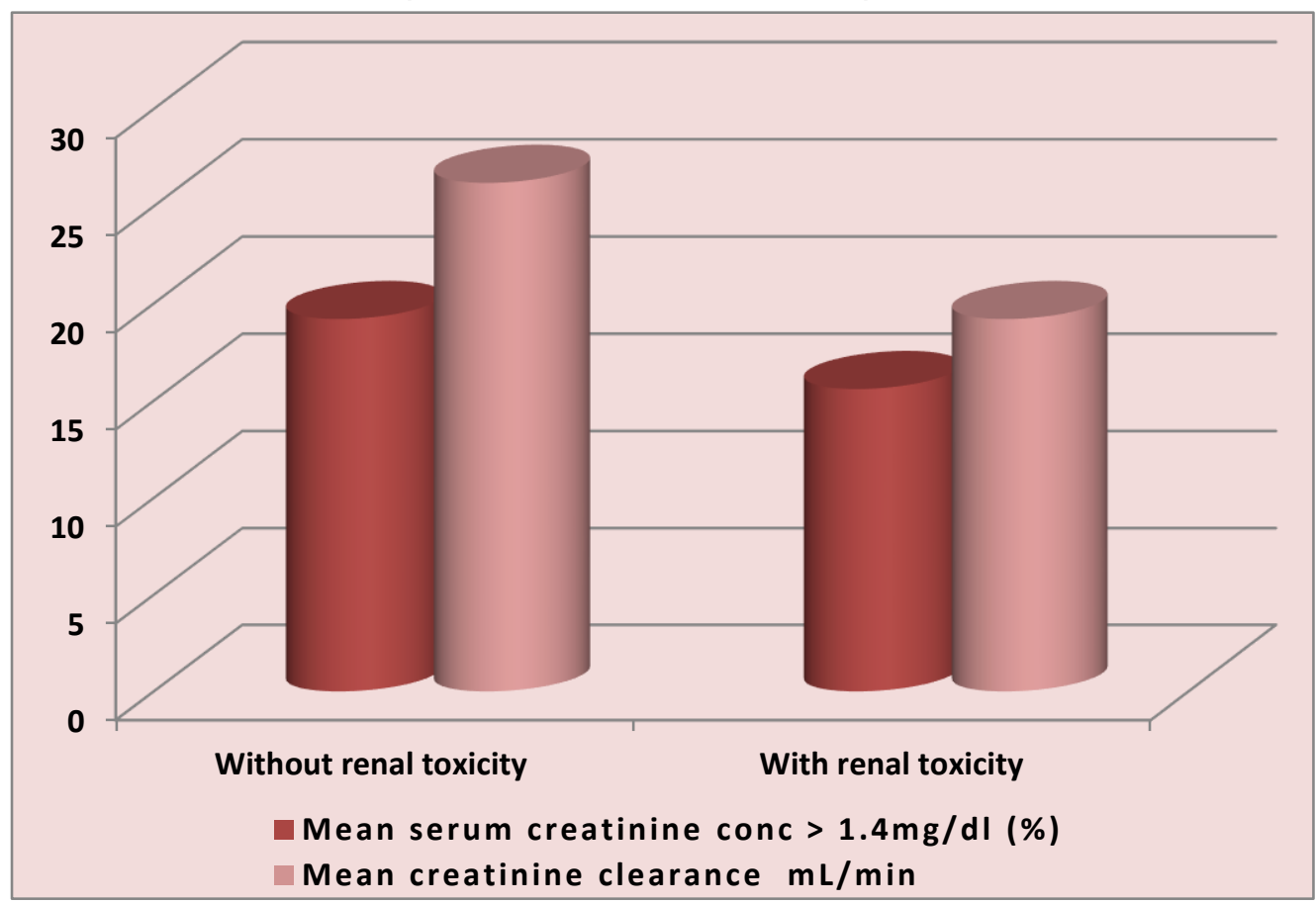

Table 2: $p$-value for the baseline renal details of the patients

\begin{tabular}{lccc}
\hline Parameter & Without renal toxicity & With renal toxicity & p-value \\
\hline Mean serum creatinine conc $\geq 1.4 \mathrm{mg} / \mathrm{dl}(\%)$ & 19.2 & 15.6 & 0.777 \\
Mean creatinine clearance $\mathrm{mL} / \mathbf{m i n}$ & 26.2 & 19.2 & 0.198 \\
\hline
\end{tabular}

Table 3: Occurrence of renal toxicity, stratified by vancomycin steady-state serum trough concentration

\begin{tabular}{lcc}
\hline Vancomycin serum trough serum conc. $(\mu \mathrm{g} / \mathrm{mL})$ & Renal toxicity $(\%)$ & p-value \\
\hline$<15$ & 28 & $0.001^{*}$ \\
$15-20$ & 31 & \\
$>20$ & 68 & \\
\hline
\end{tabular}

*: Significant

\section{DISCUSSION}

Vancomycin has been a mainstay of therapy for serious Staphylococcus aureus infections ever since its discovery in the 1950s. Although vancomycin was a second-line therapy early in its life cycle, it emerged as a first-line agent for infections due to MRSA in the 1970s. ${ }^{7}$ Over the next several decades, its usage dramatically increased due to the explosion of MRSA in both the community and health care settings. Despite the recent availability of alternative agents, vancomycin still remains the treatment of choice for serious MRSA infections. Despite its widespread use, there are growing concerns about the future role of vancomycin, particularly among patients who have invasive MRSA infections with vancomycin MICs of $1 \mathrm{mg} /$ liter. $^{8}$ Although host- and pathogen-related factors have been implicated as a cause, suboptimal vancomycin dosing has been suggested as an alternative explanation for the poorer outcomes among these patients. ${ }^{9}$ Hence; we retrospectively analyzed the rate of renal toxicity in patients treated with vancomycin for MRSA health careassociated pneumonia (HCAP) confirmed by bronchoalveolar lavage (BAL) cultures.

In the present study, we observed that aggressive vancomycin dosing, as determined by maximum measured vancomycin serum trough concentrations $\geq 15 \mu \mathrm{g} / \mathrm{mL}$, and prolonged vancomycin administration may be associated with renal toxicity among patients treated for MRSA HCAP. Jeffres et al investigated whether more aggressive vancomycin dosing is associated with greater risk for renal toxicity in patients with health careassociated pneumonia (HCAP) attributed to methicillin-resistant Staphylococcus aureus (MRSA). They retrospectively assessed the information about demographic, clinical and microbial details of the patients in a tertiary health care centre and observed that 
94 patients and concluded that aggressive vancomycin dosing and prolonged vancomycin administration may be associated with greater risk for renal toxicity in patients with MRSA HCAP. ${ }^{10}$ Hermsen et al evaluated the relationship of serum vancomycin trough concentration with clinical outcomes and nephrotoxicity for patients with deep-seated MRSA infection. They retrospectively analyzed adults with MRSA pneumonia, endocarditis or osteomyelitis who received vancomycin for $>$ or $=5$ days from June 2005 to June 2007. Patients were stratified by mean vancomycin trough level. Outcomes were clinical response, mortality, length of stay and nephrotoxicity. From the results, they concluded that clinical outcomes did not differ significantly between high and low trough groups for deep-seated MRSA infections. Nephrotoxicity was consistently higher in the high trough group, regardless of the definition used. ${ }^{11}$ Cano et al assessed the incidence of nephrotoxicity and associated risk factors in intensive care unit patients who received vancomycin for the treatment of HAP, VAP, and HCAP. They retrospectively analyzed data from a multicenter, observational study of pneumonia patients. Antibiotic-associated nephrotoxicity was defined as either an increase in serum creatinine $\geq 0.5 \mathrm{mg} / \mathrm{dL}$ or $50 \%$ above baseline, from initiation of vancomycin to 72 hours after completion of therapy. Univariate and multivariate logistic regression analyses were performed to identify risk factors for development of renal dysfunction. From the results, they concluded that Nephrotoxicity may be common among intensive care unit patients with pneumonia treated with broad-spectrum antibiotic therapy that includes vancomycin. ${ }^{12}$ Jeffres et al investigated whether vancomycin pharmacokinetic indexes (eg, serum trough concentrations or area under the concentration curve $[A \cup C]$ values) were associated with mortality for patients with health-care-associated pneumonia (HCAP) attributed to methicillin-resistant Staphylococcus aureus (MRSA). They retrospectively analyzed adult patients requiring hospitalization who were identified as having HCAP attributed to MRSA by BAL semi-quantitative cultures. From the results, they concluded that no evidence was observed which could prove that greater vancomycin trough concentrations or AUC values correlated with hospital outcome. ${ }^{13}$

\section{CONCLUSION}

From the above results, the authors conclude that among patients treated for MRSA HCAP, aggressive vancomycin dosing and prolonged administration of vancomycin may be associated with renal toxicity.

\section{REFERENCES}

1. Rybak MJ, Albrecht LM, Boike SC, Chandrasekar PH. Nephrotoxicity of vancomycin, alone and with aminoglycoside. J Antimicrob Chemother. 1990;25:679-687.

2. Downs NJ, Neihart RE, Dolezal JM, Hodges GR. Mild nephrotoxicity associated with vancomycin use. Arch Intern Med. 1989;149:1777-1781.

3. Elting LS, Rubenstein EB, Kurtin D, et al. Mississippi mud in the 1990s: Risks and outcomes of vancomycin-associated toxicity in general oncology practice. Cancer. 1998;83:2597- 2607.
4. Merten GJ, Burgess WP, Gray LV, et al. Prevention of contrastinduced nephropathy with sodium bicarbonate: A randomized controlled trial. JAMA. 2004;291:2328- 2334.

5. Moellering RC Jr, Krogstad DJ, Greenblatt DJ. Vancomycin therapy in patients with impaired renal function: A nomogram for dosage. Ann Intern Med. 1981;94:343-346.

6. Concato J, Feinstein AR, Holford TR. The risk of determining risk with multivariable models. Ann Intern Med. 1993;118:201210.

7. Rybak MJ. 2006. The pharmacokinetic and pharmacodynamic properties of vancomycin. Clin. Infect. Dis. 42(Suppl 1):S35-S39.

8. Hidayat LK, Hsu DI, Quist R, Shriner KA, Wong-Beringer A. 2006. High-dose vancomycin therapy for methicillin-resistant Staphylococcus aureus infections: efficacy and toxicity. Arch. Intern. Med. 166:2138- 2144

9. Ingram PR, Lye DC, Tambyah PA, Goh WP, Tam VH, Fisher DA. 2008. Risk factors for nephrotoxicity associated with continuous vancomycin infusion in outpatient parenteral antibiotic therapy. J. Antimicrob. Chemother. 62:168 -171.

10. Jeffres MN, Isakow W, Doherty JA, Micek ST, Kollef MH. A retrospective analysis of possible renal toxicity associated with vancomycin in patients with health care-associated methicillinresistant Staphylococcus aureus pneumonia. Clin Ther. 2007 Jun;29(6):1107-15.

11. Hermsen ED, Hanson M, Sankaranarayanan J, Stoner JA, Florescu MC, Rupp ME. Clinical outcomes and nephrotoxicity associated with vancomycin trough concentrations during treatment of deep-seated infections. Expert Opin Drug Saf. 2010 Jan;9(1):9-14

12. Cano EL, Haque NZ, Welch VL, Cely CM, Peyrani $P$, Scerpella EG, Ford KD, Zervos MJ, Ramirez JA, Kett DH. Incidence of nephrotoxicity and association with vancomycin use in intensive care unit patients with pneumonia: retrospective analysis of the IMPACT-HAP Database. Clin Ther. 2012 Jan;34(1):149-57.

13. Jeffres MN, Isakow W, Doherty JA, McKinnon PS, Ritchie DJ, Micek ST, Kollef MH. Predictors of mortality for methicillinresistant Staphylococcus aureus health-care-associated pneumonia: specific evaluation of vancomycin pharmacokinetic indices. Chest. 2006 Oct;130(4):947-55.

\section{Source of Support: Nil. Conflict of Interest: None Declared.}

Copyright: (c) the author(s) and publisher. IJMRP is an official publication of Ibn Sina Academy of Medieval Medicine \& Sciences, registered in 2001 under Indian Trusts Act, 1882.

This is an open access article distributed under the terms of the Creative Commons Attribution Non-commercial License, which permits unrestricted non-commercial use, distribution, and reproduction in any medium, provided the original work is properly cited.

Cite this article as: Anand Kumar Singh, Poonam Verma. Assessment of Renal Toxicity Associated with Vancomycin in Patients with Health Care-Associated Methicillin-Resistant Staphylococcus Aureus Pneumonia: A Retrospective Analysis. Int J Med Res Prof. 2016; 2(5):192-95. 